\section{Repeatability and reproducibility of the BVI ultrasonic Pachymeter}

P Gunvant ${ }^{1}$, DC Broadway ${ }^{2}$ and RJ Watkins ${ }^{1,2}$

\begin{abstract}
Purpose To evaluate the interobserver and intraobserver reliability of a commercially available, portable, ultrasonic pachymeter. Methods For the interobserver variability study, 42 healthy subjects underwent repeated ultrasonic pachymetry under topical anaesthesia performed by two observers. For the intraobserver study, another 30 further healthy subjects underwent repeated pachymetry by one of the observers. Agreement was analysed by means of Bland-Altman plots and by determination of the intraclass correlation coefficient. Results For the interobserver variability study, the mean measurement difference between observers was $0.7 \mu \mathrm{m}(95 \% \mathrm{CI}-0.8-2.2 \mu \mathrm{m})$ and the intraclass correlation coefficient was 0.9958 (95\% CI 0.9922-0.9977). The Bland-Altman plot showed narrow limits of agreement with respect to central corneal thickness (CCT). For the intraobserver variability study, the mean difference between the repeated measurements was $0.9 \mu \mathrm{m}(95 \% \mathrm{CI}-3.1-1.3 \mu \mathrm{m})$. The intraclass correlation coefficient was $0.9934(95 \%$ CI 0.9863-0.9969). A Bland-Altman plot again showed narrow limits of agreement with respect to CCT. The mean CCT for 72 subjects was $538 \mu \mathrm{m}(95 \%$ CI $528-545 \mu \mathrm{m})$.

Conclusions Measurements of CCT using the BVI Pocket Pachymeter were repeatable and had excellent interobserver reliability.

Measurement variation amounted to less than $0.2 \%$ assuming a mean CCT of $538 \boldsymbol{\mu m}$.

Eye (2003) 17, 825-828. doi:10.1038/

sj.eye. 6700485
\end{abstract}

Keywords: cornea; pachymetry; ultrasound; validity

\section{Introduction}

Refractive surgery, the management of corneal disease, contact lens problems, and the postoperative management of penetrating keratoplasty have made accurate measurement of corneal thickness of prime concern. ${ }^{1}$ One could now add to this list the management of patients with glaucoma and ocular hypertension..$^{2-4}$ It has been known for some time that interindividual variation in central corneal thickness (CCT) influences intraocular pressure (IOP) measurement. ${ }^{5-9}$ Orssengo and Pye $^{10}$ using an engineering approach to the cornea have developed a tabulated algorithm for calculating a 'true' IOP. The process modifies a Goldmann tonometry reading, taking into account CCT and, to a lesser extent, corneal curvature. The algorithm requires a mean CCT to be calculated in the first instance.

The process of measuring CCT (pachymetry) can be carried out optically (including specular microscopy ${ }^{11}$ ) and optical coherence tomography ${ }^{12}$ or by using ultrasound. Thornton $^{1}$ established that certain ultrasonic pachymeters are reproducible with a low degree of bias, and with little interobserver variation.

Disadvantages of ultrasonic pachymetry are the requirement for a topical anaesthetic and the potential for corneal injury caused by repeated contact with the ultrasonic probe. However, ultrasonic pachymetry has become the favoured mode of CCT measurement in clinical practice. This is because of the technical problems encountered with optical pachymeters, which suffer from poor intersession repeatability, significant interobserver variation, and significant misalignment-induced overestimation of CCT in the left eye, coupled with the expensive and, to some extent, unwieldy nature of specular microscopy. ${ }^{13}$ A further problem with specular microscopy relates to the inability of some specular microscopes to obtain readings on oedematous or opaque corneas. ${ }^{14}$ On the other hand, Muscat et $a l^{12}$ have shown that using an optical coherence tomography system designed for retinal thickness measurement allows reliable,
${ }^{1}$ Department of Optometry and Ophthalmic Dispensing Anglia Polytechnic University

Cambridge, UK

${ }^{2}$ Department of Ophthalmology Norfolk and Norwich University Hospital

NHS Trust

Norwich, UK

Correspondence:

P Gunvant

Department of Optometry and Ophthalmic Dispensing Anglia Polytechnic

University

East Road

Cambridge CB1 1PT, UK

Tel: +441223 363271

x 2684

Fax: + 441223417712

Email:contact@

pinakin-gunvant.com

Received: 8 July 2002 Accepted in revised form: 17 December 2002

Competing interests: none 
reproducible pachymetry after minor modifications to the system. The latter technique also has the advantage of being noncontact. A recent study ${ }^{15}$ showed that the Orbscan topography machine was the most repeatable instrument, compared to ultrasound and optical pachymeters.

The BVI Pocket Pachymeter (BV International, Clermont-Ferrand, France) is a commercially available ultrasonic pachymeter. The purpose of the present study was to investigate the inter- and intraobserver variability of this instrument with a view to using the pachymetric measurements, both in a clinical setting and in research on subjects with glaucoma.

\section{Materials and methods}

The BVI Pachymeter consists of a portable, batteryoperated processing unit to which is attached a control foot pedal and an ultrasonic probe. The probe had a frequency of $20 \mathrm{MHz}$ and the default velocity (of sound through the cornea) of $1620 \mathrm{~m} \mathrm{~s}^{-1}$ was used. The ultrasonic probe automatically measured the time between the two echoes from the anterior and posterior surfaces of the cornea, and from this is calculated a CCT value, which was displayed on the unit's liquid crystal display. The BVI Pachymeter is also able to measure peripheral corneal thickness, but this feature was not used in this study.

Benoxinate hydrochloride $0.4 \%$ was used in all cases for topical anaesthesia and a series of readings on a subject took no longer than $20 \mathrm{~s}$. Subjects were seated and asked to fixate on a distant target. The measurement technique involved lightly placing the hand-held probe on the cornea. An automatic reading was taken by the pachymeter after being primed by depressing the foot pedal. A short 'beep' indicated a successful measurement. The instrument requires adequate corneal contact for a successful measurement. If the probe is misaligned by more than $10^{\circ}$, then the instrument does not take a measurement. The individual observer judged the probe alignment and placement on the cornea. For central measurements, the subject's pupil was used as an anatomical landmark for the purposes of alignment. Using the pupil as an anatomical landmark ensures an identifiable zone for consistent measurements. It has to be noted that this may not be the thinnest region of the cornea. Furthermore, where the pupil is eccentric, measurements may not be representative of the central cornea.

In both experiments, in order to obtain the CCT for a subject, 10 measurements were taken and the smallest value, as long as it was consistent with other values, was recorded as the CCT. The choice of the lowest value was considered preferable to using the mean of a series of readings since the smallest value was considered more likely to indicate perpendicular placement of the probe on the cornea. Larger values might have indicated angled probe placement (although less than $\left.10^{\circ}\right)^{5}$ or a slightly off-centre placement of the probe. The smallest measurement was recorded by an assistant with the observer unaware of the measurement values.

The experiments were approved by the Norfolk and Norwich District Ethics Committee and the Anglia Polytechnic University Departmental Ethics Committee. Written informed consent was obtained from each subject and the tenets of the Declaration of Helsinki were observed in both experiments.

\section{Interobserver variability}

In all, 42 subjects underwent pachymetry, as described above, performed by two observers who were experienced in the use of the instrument. Subjects were recruited from the staff of the Department of Ophthalmology, Norfolk and Norwich University Hospital NHS Trust. One eye of each subject was chosen randomly (25 right eyes and 17 left eyes). In all, 29 subjects were female and 13 were male. The median age of the subjects was 29 years (range 20-60 years), median spherical refraction equivalent was $0.00 \mathrm{D}$ (range from +3.00 to $-6.00 \mathrm{D}$ ), and none had slit-lamp

biomicroscopic evidence of corneal disease. None of the subjects examined in the study had astigmatism of greater than 1.5D. The order in which observer 1 and observer 2 took the measurements was randomised. The interval between observers performing the measurements was no longer than $5 \mathrm{~min}$.

\section{Intraobserver variability}

Observer 1 performed pachymetry, as described above, on a different group of 30 subjects, recruited from staff and students at Anglia Polytechnic University. The same observer repeated this after an interval of no longer than $5 \mathrm{~min}$. One eye of each subject was chosen randomly (13 right eyes and 17 left eyes). In all, 20 subjects were female and 10 were male. The median age of the subjects was 22 years (range 19-55 years), median spherical refraction equivalent was $0.00 \mathrm{D}$ (range +1.00 to $-3.75 \mathrm{D})$, and none had slit-lamp biomicroscopic evidence of corneal disease.

All statistical analyses (except the intraclass correlation coefficient) were performed using Analyse-It Software v.1.62 for Microsoft Excel (Analyse-It Software, Leeds, UK). An intraclass correlation coefficient was calculated using SPSS v. 9.0 (SPSS Inc., Chicago, IL, USA). 


\section{Results}

\section{Interobserver variability}

For observer 1, the mean CCT for the 42 subjects was $538 \mu \mathrm{m}(95 \%$ CI $526-549 \mu \mathrm{m})$ and for observer 2 was $539 \mu \mathrm{m}(95 \%$ CI $527-550 \mu \mathrm{m})$. The mean measurement difference between observers was $0.7 \mu \mathrm{m}(95 \% \mathrm{CI}-0.8$ $2.2 \mu \mathrm{m})$. To assess agreement between both observers, Bland-Altman plot was constructed (Figure 1). It can be seen that the limits of agreement are narrow with respect to the mean CCT. To further assess agreement, the intraclass correlation coefficient was calculated and found to be 0.9958 (95\% CI 0.9922-0.9977). The analysis indicated excellent agreement.

There was a significant relation between interobserver measurement difference and refractive error (Kendall's $\tau=0.27 ; P=0.0243$ ), but an outlier heavily influenced this result. Removal of this data point made the relation nonsignificant (Kendall's $\tau=0.17 ; P=0.1776$ ). Subject gender, eye laterality, and age did not influence the magnitude of the interobserver difference.

\section{Intraobserver variability}

The mean baseline CCT for 30 subjects measured by observer 1 was $535 \mu \mathrm{m}$ and for the second measurement was $534 \mu \mathrm{m}$. The mean difference between the first and second measurements was $0.9 \mu \mathrm{m}(95 \% \mathrm{CI}-3.1-1.3 \mu \mathrm{m})$. A Bland-Altman plot (Figure 2) showed again that in this case, the limits of agreement were narrow with respect to the mean CCT, although the confidence intervals were slightly wider than those in Figure 1. To further assess the agreement, the intraclass correlation coefficient was calculated and found to be 0.9934 (95\% CI 0.9863-0.9969). The analysis indicated excellent agreement. There was no

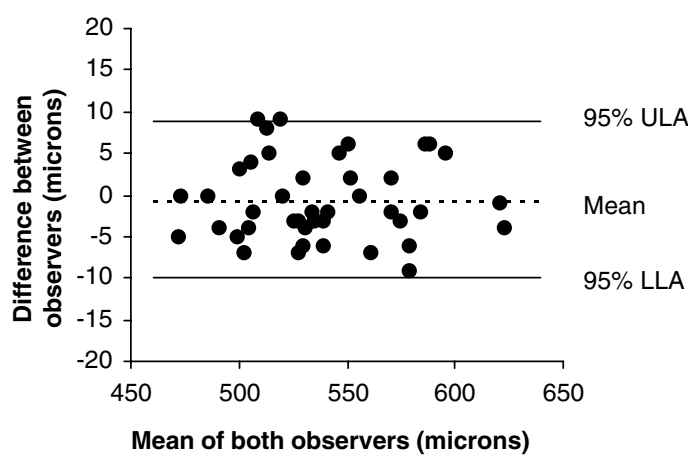

Figure 1 A Bland-Altman plot of data from the interoperator reproducibility study. Mean CCT each subject was plotted against the difference in CCT measurements between observers 1 and 2. ULA: upper limit of agreement; LLA: lower limit of agreement. All data points $(n=42)$ lie at or within 2 SDs of the mean, see text for details.

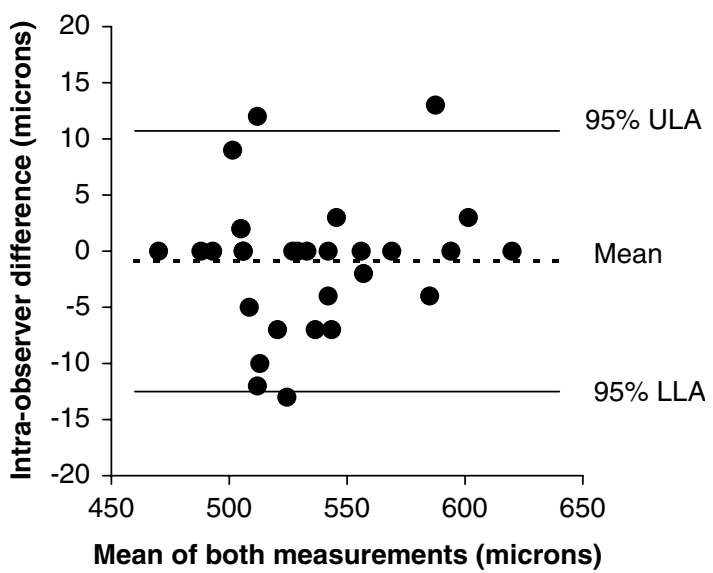

Figure 2 A Bland-Altman plot of data from the intraoperator reproducibility study. Mean central corneal thickness for each subject was plotted against the difference in CCT between two measurements obtained by the same observer. ULA: upper limit of agreement; LLA: lower limit of agreement. All but three of the data points $(n=30)$ lie at or within 2 SDs of the mean, see text for details.

significant relation between intraobserver measurement difference and refractive error. Subject gender, eye laterality, and age did not influence the magnitude of the intraobserver difference.

\section{Discussion}

This study demonstrated that in common with other ultrasonic pachymeters, measurement of CCT using the BVI Pocket Pachymeter is repeatable and has excellent interobserver reliability. The unit was easy to operate and the technique required minimal patient cooperation.

The mean interobserver difference in CCT measurement that we found was $0.7 \mu \mathrm{m}$, which compared favourably with that obtained by Gordon et $a l^{16}$ who used a different ultrasonic pachymeter. Furthermore, the intraobserver level of agreement was excellent with a mean difference between measurements of only $0.9 \mu \mathrm{m}$. This was an excellent level of agreement amounting to a CCT variation of only around $0.17 \%$, assuming an average CCT of $538 \mu \mathrm{m}$.

Small variations in CCT measurement may arise from technical aspects of the use of the BVI Pachymeter such as differing forces of probe placement by different observers and slightly off-centre probe placement (the probe was hand held and had no internal fixation light). The previously demonstrated diurnal variation of $\mathrm{CCT}^{17,18}$ would not have been a factor in our study because the time interval between measurements was short. 
As with all ultrasonic pachymeters, an estimate of corneal thickness is all that is obtainable with the BVI instrument. The CCT measurement depends on the calibration velocity of the instrument, probe frequency and, of course, the normal interindividual variations of corneal structure and physiology, especially hydration. ${ }^{18}$

The variability in the estimated CCT between different pachymeters is well known. ${ }^{1,14,16,19}$ Even among ultrasonic pachymeters, using the same calibration ultrasonic velocity, there may be a large degree of variability..$^{19}$ Owing to the increasing number of refractive surgical procedures being carried out, the absolute value of an individual's CCT is becoming important. The BVI Pachymeter has a utility called 'Bias correction' which can be used to correct any known errors in measurement. This can be programmed to correct the measured values by either a known amount or percentage. For example, if the pachymeter is known to give consistently higher or lower values than another pachymeter, for instance, an optical coherence tomograph, then using the bias correction, will adjust the measured values obtained with the BVI Pachymeter. We conclude that when used in the manner described above, the BVI Pocket Pachymeter shows excellent inter- and intraobserver reliability.

\section{Acknowledgements}

We thank the volunteers who acted as subjects in our study.

\section{References}

1 Thornton SP. A guide to pachymeters. Ophthalmic Surg 1984; 15(12): 993-995.

2 Gordon MO, Beiser JA, Brandt JD, Heuer DK, Higginbotham EJ, Johnson CA et al. The Ocular Hypertension Treatment Study: baseline factors that predict the onset of primary open-angle glaucoma. Arch Ophthalmol 2002; 120(6): 714-720; discussion 829-830.

3 Kass MA, Heuer DK, Higginbotham EJ, Johnson CA, Keltner JL, Miller JP et al. The Ocular Hypertension Treatment Study: a randomized trial determines that topical ocular hypotensive medication delays or prevents the onset of primary open-angle glaucoma. Arch Ophthalmol 2002; 120(6): 701-713; discussion 829-830.
4 Palmberg P. Answers from the ocular hypertension treatment study. Arch Ophthalmol 2002; 120(6): 829-830.

5 Copt RP, Thomas R, Mermoud A. Corneal thickness in ocular hypertension, primary open-angle glaucoma, and normal tension glaucoma. Arch Ophthalmol 1999; 117(1): 14-16.

6 Ehlers N, Bramsen T, Sperling S. Applanation tonometry and central corneal thickness. Acta Ophthalmol (Copenh) 1975; 53(1): 34-43.

7 Johnson M, Kass MA, Moses RA, Grodzki WJ. Increased corneal thickness simulating elevated intraocular pressure. Arch Ophthalmol 1978; 96(4): 664-665.

8 Shah S, Chatterjee A, Mathai M, Kelly SP, Kwartz J, Henson $\mathrm{D}$ et al. Relationship between corneal thickness and measured intraocular pressure in a general ophthalmology clinic. Ophthalmology 1999; 106(11): 2154-2160.

9 Stodtmeister R. Applanation tonometry and correction according to corneal thickness. Acta Ophthalmol Scand 1998; 76(3): 319-324.

10 Orssengo GJ, Pye DC. Determination of the true intraocular pressure and modulus of elasticity of the human cornea in vivo. Bull of Mathmatical Biol 1999; 61: 551-572.

11 Wheeler NC, Morantes CM, Kristensen RM, Pettit TH, Lee DA. Reliability coefficients of three corneal pachymeters. Am J Ophthalmol 1992; 113(6): 645-651.

12 Muscat S, McKay N, Parks S, Kemp E, Keating D. Repeatability and reproducibility of corneal thickness measurements by optical coherence tomography. Invest Ophthalmol Vis Sci 2002; 43(6): 1791-1795.

13 Salz JJ, Azen SP, Berstein J, Caroline P, Villasenor RA, Schanzlin DJ. Evaluation and comparison of sources of variability in the measurement of corneal thickness with ultrasonic and optical pachymeters. Ophthalmic Surg 1983; 14(9): 750-754.

14 Bovelle R, Kaufman SC, Thompson HW, Hamano H. Corneal thickness measurements with the Topcon SP-2000P specular microscope and an ultrasound pachymeter. Arch Ophthalmol 1999; 117(7): 868-870.

15 Marsich MW, Bullimore MA. The repeatability of corneal thickness measures. Cornea 2000; 19(6): 792-795.

16 Gordon A, Boggess EA, Molinari JF. Variability of ultrasonic pachometry. Optom Vis Sci 1990; 67(3): 162-165.

17 Harper CL, Boulton ME, Bennett D, Marcyniuk B, JarvisEvans JH, Tullo AB et al. Diurnal variations in human corneal thickness. Br J Ophthalmol 1996; 80(12): 1068-1072.

18 Lattimore Jr MR, Kaupp S, Schallhorn S, Lewis RT. Orbscan pachymetry: implications of a repeated measures and diurnal variation analysis. Ophthalmology 1999; 106(5): 977-981.

19 Reader III AL, Salz JJ. Differences among ultrasonic pachymeters in measuring corneal thickness. J Refract Surg 1987; 3: 7-11. 